\section{Detecting the Fetal Heart}

In exploring the quiet yet turbulent world of the human gravid uterus, ultrasound has an increasingly important place, largely owing to the unflagging efforts of Professor I. Donald, of Glasgow. Now from his unit comes another advance, described by Dr. H. P. Robinson at page 466 in this issue of the B.M.F. By means of it the fetal heart can be "heard" as early as the 48th day of menstrual age. This means about 7 weeks gestational age, which is most remarkable.

Embryological evidence suggests that the heart assumes its pumping action at the end of the third week of gestational age ${ }^{1}$ and that the major blood vessels are in active use by the sixth week. To be able to detect cardiac activity in the intact embryo at all by the seventh week is therefore astonishing enough, but to have this knowledge available for clinical use right from the start is amazing. British science is often upbraided for delay between discovery and application, but here the two are simultaneous. The fetal heart had been "heard" with a vaginal transducer at the eighth week of pregnancy (menstrual age) by $P$. Jouppila, ${ }^{2}$ but the advantage of Robinson's method is that the pulsed ultrasound is applied through the abdominal wall with scarcely any upset to the patient.

For many years it was held that the only certain evidence of pregnancy was an $x$-ray picture showing fetal parts or hearing the fetal heart on auscultation. $X$-rays do not show the fetal skeleton until 16 weeks menstrual age, and the heart cannot literally be heard until about the 24th week. The advent of portable ultrasound equipment using the Doppler effect to detect the fetal heart lowered the time of "hearing" the heart to about 12 to 14 weeks, and is now in use in many obstetric departments. It has been a most useful piece of apparatus, since a common problem is to know for sure whether a fetus is alive or dead, and this can often now be solved in a matter of minutes at the bedside. Though Robinson's technique is more complex than this, it is certainly not impossibly complicated, for the heart can be detected in under five minutes and usually in less than two.

In England and Wales there are about 750,000 births every year. It is said that about $10 \%$ of all pregnancies are complicated by threatened and missed abortions, giving a number probably well in excess of 100,000 per year. Many of these patients have to be kept in bed waiting for events to declare themselves. This new technique should cut down the anxious delay in making decisions about whether to continue with conservative treatment or to empty the uterus.

Fears are sometimes expressed that ultrasound may cause fetal damage. There is no evidence of this. Newborn babies who have been subject to it in utero show no greater incidence of abnormality than controls. ${ }^{34}$ Chromosomal damage has been sought in embryos insonated before hysterotomy ${ }^{5}$ and in lymphocyte cultures exposed to ultrasound. ${ }^{6} 7$ Despite some conflicting exper:mental results the weight of the evidence and the generally accepted view are that it is harmless, and there seems to be no good reason why this new technique for the early detection of the fetal heart should come under a cloud.

Ultrasound was first used by the navy towards the end of the 1914-18 war in the asdic detector (Antisubmarine Detection and Investigation Committee). Power ultrasound was developed from this for detecting flaws in metals and for cleaning materials. In a much attenuated form we now have sonar (Sound Navigation and Ranging), which ought to be differentiated from power ultrasound, since diagnostic ultrasound is of comparatively very low energy. Sonar has an extensive range of usefulness in obstetrics and gynaecology as well as other disciplines. It can be used in the diagnosis of early pregnancy, abortion, blighted ovum, twins long before other methods can compete, life or death of the embryo, hydatidiform mole, and pelvic tumours. It can localize the placenta and monitor fetal growth. There is now no longer any need to justify its use and it ought to be widely available to all obstetric and gynaecological departments, for it brings a new dimension to diagnosis and management which cannot be ignored.

1 Johnson, J. W. C., in Intra-uterine Development, ed. A. C. Barnes, p.

176. London, Henry Kimpton, 1968.
2 Jouppila, P., Acta Obstetrica et Gynecologica Scandinavica, 1971, 50, Suppl. 15, 3 .

3 Hellman, L. M., Duffus, G. M., Donald, I., and Sunden, B., Lancet, $1970,1,1133$.

4 Lucas, M., Mullarkey, M., and Abdulla, U., British Medical fournal, 1972, 3, 795.

5 Abdulla, U., et al., Lancet, 1971, 2, 829.

Abdulla, U., Talbert, D., Lucas, M., and Mullarkey, M., British Medical fournal, 1972, 3, 797.

7 Boyd, E., et al., British Medical fournal, 1971, 2, 501.

\section{Chest Colds}

Some colds "go down to the chest," as everyone knows. Bronchitics and asthmatics are especially aware of the sequence, and their ventilatory impairment can be measured by simple spirometry. ${ }^{1}$ Often the impairment persists for several weeks, which is in keeping with clinical observation. But the usual tests of lung function are insufficiently sensitive to detect pulmonary changes except when postcoryzal bronchitis or pneumonitis develops. Recent studies suggest that measurements of dynamic compliance may provide the only evidence that the lungs are involved.

Dynamic compliance (Cdyn) is estimated from simultaneous measurements of tidal volume and intrathoracic pressure at increasing rates of respiration. Normally the readings fall by no more than about $20 \%$ of the values obtained under static conditions. In patients with airways obstruction the dynamic compliance falls much more than this owing to regional differences in the speed with which different parts of the lung can expand and contract. This abnormal fall may be used as an index of increased resistance to flow in the distal airways when the range of regional differences is at least four times the normal, provided that both the resistance of the central airways measured by the body plethysmograph and the elastic recoil pressure of the lungs are normal. ${ }^{2}$

J. J. Picken and colleagues ${ }^{3}$ have performed physiological tests on 12 healthy young adults during and two months after acute coryza. No changes were found in the vital capacity, expiratory flow rates, thoracic gas volume (Vtg), airways resistance (Raw), or Vtg $\times$ Raw. Static compliance (Cstat) was normal in all but one, who showed a variable pattern. When they compared dynamic compliance at increasing breathing frequency with static compliance, they noted a reduction in four of the 12 patients, which persisted two months later. In the face of normal results in the other tests these changes in dynamic compliance may properly be taken as evidence of 
small airways disease during and after acute respiratory infections.

What happens when viral infections reach the smaller bronchi and bronchioles is by no means certain. Histopathological material is not available in man, nor has a suitable animal host been found which reacts to viruses in a similar way. Increased muscular tone does not seem to be important, since bronchodilators have no effect on the impaired dynamic compliance. ${ }^{3}$ Inflammatory changes with increased secretions in the airways seem to be most likely, possibly associated with local patchy collapse, but they cannot be extensive when the vital capacity and static compliance are normal. Some support for this view comes from the observation that when radioactive labelled particles penetrate the small airways they may persist for as long as six weeks after acute coryza. ${ }^{4}$ Another explanation, which would fit the physiological findings, is the possibility of localized defects in the production of surfactant, which could upset the stability of the distal air passages. If this happens, some impairment in gaseous exchange might be expected. 5 Perhaps further investigations will prove this to be so.

Most respiratory viruses are capable of affecting the smaller airways-for example, influenza and respiratory syncytial virus-and also Mycoplasma pneumoniae. Much could be gained by studying people during and after common colds with a view to determining which viruses are most likely to involve the lungs, the circumstances in which they do, and the length of time the defects persist.

1 Angel, J. H., Fletcher, C. M., Hill, I. D., and Tinker, C. M., British Fournal of Diseases of the Chest, 1965, 59, 66.

2 Woolcock, A. J., Vincent, N. J., and Macklem, P. T., fournal of Clinical Investigation, 1969, 48, i097.

3 Picken, J. J., Niewoehner, D. E., and Chester, E. H., American fournal of Medicine, 1972, 52, 738 .

${ }^{4}$ Lourenco, R. V., Stanley, E. D., Gatmaitan, B., and Jackson, G. C., fournal of Clinical Investigation, 1971, 50, 62a.

${ }^{5}$ Mead, J., New England fournal of Medicine, 1970, 282, 1318.

\section{The Elderly in Scottish Hospitals}

The hospital services required for persons aged 65 and over have recently been evaluated by a study of their use in Scotland. ${ }^{1}$ The elderly constituted $11.7 \%$ of the total population of Scotland in 1968, and this proportion is expected to rise to $13.1 \%$ in 1978 and to fall to $12.8 \%$ in 1988. A sharp increase in people of 75 and over is predicted, and they are greater users of hospital beds than the more recently retired. Consequently some 3,000 additional beds may be needed in non-psychiatric hospitals alone in the next 20 years specially to care for the needs of the elderly. Nearly $90 \%$ of the additional beds $(2,600)$ will be required for the care of the people aged 75 and over, 1,900 being for elderly women and 700 for elderly men.

The classification of an elderly patient as needing hospital care carries a social as well as a medical diagnosis. Marriage, for instance, is a powerful safeguard against admission to hospital. The lowest rate of hospital residence is among married elderly men and women aged 65-69 and the highest is among single elderly men and women aged 85 and over, with the women in the lead. Persons aged 85 and over are some 10 to 14 times more likely to be resident in hospital than a married person of 65-69. Married people in hospital remain for a shorter time than either the single or the widowed or divorced elderly. Most elderly people admitted to hospital are discharged within a very short time, but a small proportion remain for long spells. The mean duration of stay of 66 days for patients discharged from geriatric assessment units suggests that some patients were remaining in these beds for want of suitable alternative accommodation.

In 1966 excessive or inappropriate use of beds was found to have occurred in nearly $17 \%$ af all bed days in the acute specialties. This argues, firstly, for an increase in the supply of long-stay accommodation, and, secondly, for a reclassification of certain beds at present in acute specialties, or for a combination of the two. The main excess of bed use was attributed to patients aged 15-64 years (58\% of total bed use). This is an interesting finding in view of the fact that it is the blockage of beds by elderly people ( $7 \%$ of total bed use) which has received the bulk of publicity hitherto. Elderly women occupy short-stay beds inappropriately more than elderly men. About one-sixth of the total bed days spent in acute hospitals in 1966 involved patients of whom most would probably have been cared for outside acute wards if alternative accommodation had been available. Some of the elderly would more appropriately have been looked after in residential homes or possibly at home if suitable help had been available.

A notable feature of this study is the long average length of stay of patients in mental hospitals. On average an old person discharged from a mental hospital had spent over 50 times as long there as his counterpart treated in an acute unit in a general hospital and over seven times as long as one from a geriatric unit. The average acute elderly patient discharged from hospital spent over three weeks in hospital after admission, the average geriatric patient over five months, while the average mental hospital elderly patient spent well over three years in hospital.

When discharges from mental hospitals in 1966 are examined, comparison between England and Wales and Scotland shows that the elderly in Scotland are more likely to be admitted to a mental hospital, and once admitted there they remain longer. In general, blockage of beds by the elderly is lower in England and Wales than in Scotland, and this could be explained only by a comprehensive assessment of traditions in care, relative levels of community and social provision, housing, patterns of morbidity, and other such factors. But it does seem possible that Scottish hospitals, both mental and non-psychiatric, exercise a more important role in the social as well as the medical care of the elderly, and that every year many elderly people are admitted to hospitals in Scotland who might more appropriately have been cared for in the community if resources for their care were better. There is a relative deficiency of domiciliary provision for the elderly outside hospital in Scotland.

This report assumes the continuation of a trend towards home care by relatives, friends, and neighbours, but that will take place only if the social services are expanded. A point not stressed is the high incidence of senile dementia in the very age group ( 75 years and over) in which the expected increase in numbers of the elderly will be greatest. Thus it is evident that more protected housing and alternative accommodation such as homes for the physically and mentally frail are now required and that hospital outpatient facilities for the elderly should be increased.

1 Downie, B. N., The Elderly in Scottish Hospitals 1961-1966. Scottish Health Service Studies No. 21. Scottish Home and Health Department, 1972. 